\title{
АСТРОФИЗИКА
}

\author{
Э. В. Серга
}

\section{О ПРИРОДЕ КРАСНЫХ СМЕЩЕНИЙ В СПЕКТРАХ ГАЛАКТИК}

\begin{abstract}
Аннотация. Предмет исследования - феномен красных смещений в спектрах галактик. Современное естествознание связывает этот эффект с доплеровским смещением вследствие взаимного удаления галактик (расширение Вселенной). Доплеровская интерпретация красных смещений основана на представлении о космическом пространстве (космическом вакууме) как пустоте. Однако такая интерпретация является устаревшей в свете новых научных знаний, позволяющих рассматривать космический (физический) вакуум как материальную среду со специфическими свойствами. Представление о вакууме как материальной среде с собственной структурой, составом и свойствами основано на данных о вакуумных эффектах, хорошо известных в квантовой теории поля и физике конденсированных сред. Ключевой предпосылкой работы является позиция о единстве теории вакуума в физике микромира и в физике космоса. Для решения поставленных задач использованы методы формальной логики, общенаучные исследовательские подходы (обобщение, анализ, синтез теоретических положений и опытных данных). Предлагаемая модель объясняет эффект красных смещений в спектрах галактик как один из вакуумных эффектов, обусловленный взаимодействием фотонов с вакуумом как материальной средой. Такой подход позволяет на основе новых научных данных вернуться к ранее отвергнутой гипотезе известного российского и советского астрофизика А.А. Белопольского о потере энергии фотонами при их движении в космическом пространстве, выдвинутой им в 1929 году.
\end{abstract}

Ключевые слова: Космический вакуум, Красное смещение, Комбинационное рассеивание света, Космология, Старение фотонов, Расширение Вселенной, Закон Хаббла, Гипотеза Белопольского, Галактика, Квазар.

Abstract. The subject of this research is the phenomenon of redshifts in galactic spectrums. Modern natural science associates this effect with the Doppler shift as the result of the expending universe. Doppler's interpretation of the redshifts is based on the perception of the cosmic space (cosmic vacuum) as emptiness. However, such interpretation is outdated in light of modern scientific knowledge, which allows us to observe cosmic (physical) vacuum as a material environment with specific characteristics. The key prerequisite for this work is the position on the unity of the vacuum territory in physics of the microworld and space physics. The proposed model explains the effect of redshifts in galactic spectrums as one of the vacuum effects explained by the interaction of the photons with the vacuum as a physical environment. Such approach allows, using new scientific data, returning to the earlier rejected hypothesis of the Russian and Soviet astrophysicist Aristarkh Belopolsky on loss of energy of photons in their movement through space proposed by him in 1929.

Keywords: Space Vacuum, Redshift, Raman scattering, Cosmology, Tired light, Expansion of space, Hubble's law, Belopolsky's Hypothesis, Galaxy, Quasar.

\section{Введение}

войства физического вакуума как материальной среды проявляются в различных вакуумных эффектах, которые наблюдаются в микромире и космосе. В отличие от физики микромира, где эти эффекты достаточно хорошо известны и изучены, в физике космоса они исследованы в меньшей степени и не всегда имеют удовлетворительное объяснение. В ряде случаев эффекты, которые можно объяс- нить влиянием физического вакуума, интерпретируются на основе положений, не учитывающих свойства вакуума как материальной среды. Это относится, в частности, к современной интерпретации красных смещений в спектрах галактик.

В современном естествознании эффект красных смещений в спектрах галактик объясняют доплеровским смещением вследствие взаимного удаления галактик. Согласно принятой большинством специалистов теории расши- 


\section{Исследования космоса 4(5) • 2017}

ряющейся Вселенной, галактики удаляются со скоростью, определяемой законом Эдвина Хаббла (Hubble, Edwin Powell, 1989-1953). При этом предполагается, что космическое пространство лишено осязаемой материи, т.е. рассматривается как пустота. Из такого подхода следует, что у доплеровской интерпретации красных смещений нет альтернативы.

Однако, если принять позицию, что космическое пространство не является пустотой, а космический вакуум представляет собой материальную среду $[1,2]$, то красные смещения получают объяснение как вакуумный эффект, а именно, как эффект взаимодействия физического вакуума с фотонами. Тогда с учётом новых знаний о вакууме необходимо вернуться к гипотезе Аристарха Аполлоновича Белопольского (1854-1934) о потере энергии фотонами при их движении в космическом пространстве [3, с 266267]. Эта гипотеза, выдвинутая российским и советским астрофизиком в 1929 году, не получила признания как противоречащая представлениям о космическом вакууме как пустоте.

\section{Концептуальные подходы к проблеме объяснения природы красных смещений в спектрах галактик}

В основе концептуальных подходов к объяснению природы красных смещений лежат два различных представления о физической сущности космического вакуума как среды:

- пустое пространство;

- материальная среда.

История формирования представлений о среде, заполняющей мировое пространство, тесно связана с развитием науки и обобщением данных наблюдений и экспериментов. Ранее эту среду называли «эфиром», в современной терминологии используется понятие «физический вакуум». В естествознании сложилась парадоксальная ситуация в части представлений о физическом вакууме и использовании этих представлений в теоретических построениях. Этот концепт включают в теорию, если он нужен, например, для объяснения наблюдаемых вакуумных эффектов в квантовой теория поля. Однако о нём не упоминают, если, по мнению специалистов, в этом нет необходимости (напри- мер, в небесной механике и космологии). Такое положение дел во многом определяется тем, что эффекты, возникающие в микромире и в космосе, существенно отличаются, как в силу разницы в масштабе наблюдаемых объектов, так и в силу специфики используемых методов и средств. Однако очевидно, что понимание физической сущности вакуума должно быть единым, как в физике микромира, так и в физике космоса.

В настоящее время большинство специалистов считает, что красные смещения в спектрах галактик имеют доплеровскую природу. Это означает, что космический вакуум как среда рассматривается исключительно как пустое пространство. Такое представление о космической среде основано на многовековом опыте наблюдений за движением небесных тел. Планеты Солнечной системы столетиями движутся в космическом пространстве по одним и тем же орбитам, не испытывая сколько-нибудь заметного сопротивления среды, подобно движению в пустоте.

Однако представление о космической среде как пустоте противоречит распространению в ней света. Ещё в XIX веке Майкл Фарадей (Faraday, Michael, 1791-1867) ввёл понятие поля, рассматривая его как возбуждённое состояние эфира, то есть среды, заполняющей мировое пространство. Через эту среду осуществляется взаимодействие тел. Джеймс Максвелл (Maxwell, James Clerk, 1831-1879), развивая представления М.Фарадея о поле, доказал, что свет - это электромагнитные волны, которые распространяются в материальной среде [4, с. 380]. Однако свойства этой среды (эфира) были несовместимы с представлениями о свойствах обычных известных сред. Эфир обладал сочетанием противоречивых свойств: плотной упругой среды и пустоты. Свойство плотной упругой среды проявляется в распространении света. В то же время в этой среде было возможно движение тел без сопротивления. Несовмест имость физических свойств эфира со свойствами обычных изученных сред и отсутствие опытных данных, на основе которых можно было бы объяснить его наблюдаемые противоречия, не позволили учёным прошлого построить последовательную теорию эфира.

Ситуация с пониманием противоречивых свойств физического вакуума стала проясняться 
после открытия Петром Леонидовичем Капицей (1902-1984) явления сверхтекучести жидкого гелия и создания нового направления в физике - физики конденсированных сред (ФКС). Как отмечает физик-теоретик Г.Е. Воловик (род. 1946), «в ФКС появились новые субстанции, так называемые упорядоченные среды со спонтанно нарушенной симметрией. Среди них есть жидкости, которые благодаря квантовому характеру настолько противоречат обычным представлениям о жидкости, что пришлось пересмотреть традиционное отношение к жидкости, а заодно и к эфиру (вакууму), как среде, заполняющей мировое пространство. Оказалось, что новые свойства этих жидкостей подобны свойствам физического вакуума. Эти квантовые жидкости при определённой температуре испытывают фазовый переход в сверхтекучее состояние. Одно из проявлений сверхтекучести состоит в отсутствии вязкости: такая жидкость при нулевой температуре (находясь в основном, т.е. невозбуждённом состоянии) не оказывает сопротивления движущимся телам, но обладает упругостью» [5].

Таким образом, в ФКС получил объяснение феномен существования плотной упругой среды, в которой возможно движение тел без сопротивления. Открытие сверхтекучести жидкого гелия и последующие теоретические работы в области ФКС позволили возродить на новой научной основе концепцию эфира как материальной среды, заполняющей мировое пространство, от которой физики отказались в начале XX века после появления работ Альберта Эйнштейна (Einstein, Albert, 1879-1955) по специальной теории относительности. Эта неопределенность, существовавшая в начале прошлого века, во многом сохранилась и до настоящего времени.

Как известно, А. Эйнштейн в специальной теории относительности (СТО), фактически, «упразднил» эфир, указав на несовместимость созданной им теории с гипотезой эфира [6, с. 176]. В дальнейшем в общей теории относительности (ОТО) он «вернул» эфир, утверждая: «Согласно общей теории относительности, пространство немыслимо без эфира» [7, с. 689]. Однако «возвращение эфира»не привело к отказу от СТО как теории, несовместимой с гипотезой эфира.В итоге, в последующих работах, в которых А. Эйнштейн использовал ОТО для объяснения аномального смещения перигелия Меркурия и космологии, эфир не упоминается.

Статья А. Эйнштейна «Вопросы космологии и общая теория относительности» [8], опубликованная в 1917 г., была первой работой по применению новой теории к Вселенной, понимаемой как единое целое. Подходя к построению космологии, А. Эйнштейн разделял определённые общие воззрения на Вселенную. В частности, он полагал, что устройство мира должно быть простым. Вселенная как целое должна быть однородной: распределение вещества в ней всюду одинаково по плотности. Пространство также является однородным, т.е. всюду одинаковым по своим геометрическим свойствам. А. Эйнштейн исходил из того, что Вселенная пребывает в неизменном статичном состоянии. Тогда такая Вселенная симметрична относительно сдвигов во времени. Статичность связывалась с малыми скоростями движения звёзд. При этом все точки мира были равноправны: переход от одного места к другому ничего не меняет в общей геометрии. А. Эйнштейн предполагал также, что вместе с однородностью мира имеется ещё изотропия, т.е. равноправность всех направлений в пространстве. Обе эти пространственные симметрии присущи Вселенной как целому и проявляются в её крупномасштабных свойствах.

Этот образ Вселенной представлялся в то время естественным и очевидным. Но оказалось, что уравнения гравитационного поля в теории А. Эйнштейна не допускают статичности Вселенной. Тогда в целях согласования теории с данными наблюдений А. Эйнштейн решил несколько подправить уравнения. Он предположил, что наряду с обычным веществом, все частицы которого испытывают взаимное притяжение, в мире существует ещё необычная среда, создающая отталкивание. Она способна компенсировать взаимное притяжение частиц вещества. В то же время А. Эйнштейн ничего не говорит о природе источников гравитационного отталкивания. Он формально вводит в уравнения дополнительное слагаемое, которое назвал космологическим членом. В результате, дополненные таким путём уравнения допускают статичную Вселенную.

В 1922 г. Александром Александровичем Фридманом (1888-1925) были получены реше- 


\section{Исследования космоса 4(5) • 2017}

ния уравнений ОТО, кода они были применены к Вселенной в целом [9, с. 316-322]. А.А. Фридман использует уравнения А. Эйнштейна в общей форме, включая космологический член. Полученные решения допускают стационарную и нестационарную Вселенную. Поначалу А. Эйнштейн счёл результаты А.А. Фридмана подозрительными [10], но потом изменил своё отношение к ним. В частности, он писал: «Моя критика основывалась на ошибке в вычислениях. Я считаю результаты Фридмана правильными и проливающими новый свет» [11].

Таким образом, позиция А.Эйнштейна в части отношения к эфиру в период становления новой космологии была достаточно непоследовательной. Вначале, в целях согласования ОТО с положением о пространственной замкнутости Вселенной, он ввёл в уравнения космологический член, который на классическом языке означает гравитационное отталкивание. Впоследствии, после открытия Э. Хабблом красного смещения в спектрахудалённых галактик, А.Эйнштейн счёл космологический член излишним [12], хотя для этого было не больше оснований, чем при его введении.

Применительно к рассматриваемой проблеме необходимо определиться с представлением о космической среде, а именно: это пустое пространство или материальная среда? Если космическая среда - это материальная среда, то необходимо дать объяснение природы красных смещений, отличное от доплеровской интерпретации. Как отмечалось, ранее такое объяснение было дано А.А. Белопольским. Он предположил, что частота (а значит и энергия) фотонов убывает по мере их движения в пространстве, причём так, что изменение частоты пропорционально пройденному расстоянию. Как писал А.А. Белопольский, «если считать, что направление и величины движения определяются смещением спектральных линий по принципу Доплера, то нужно говорить о скоростях. Но, если мы будем рассматривать смещение спектральных линий только как таковое, то мы должны иметь дело со светом, как с таковым, т.е. с увеличением длин волн или с уменьшением частоты» [3, с. 266-267]. В подтверждение выдвинутой гипотезы он приводит данные наблюдений звёзд и туманностей (всего порядка тысячи объектов).
A.А. Белопольский не дал какого-либо развернутого физического обоснования своей гипотезы, и она не получила признания. Против неё были выдвинуты следующие возражения. Во-первых, не ясен был механизм потери энергии фотонами в космическом пространстве, которое рассматривалось как пустота. Во-вторых, по утверждению оппонентов, если бы на пути фотонов встречались частицы межзвёздной среды, то неизбежно происходило бы отклонение фотонов от первоначального направления, и тогда изображения далёких галактик казались бы размытыми. Но наблюдения показывают, что очертания далёких галактик столь же отчётливы, как и ближайших к нам галактик.

В настоящее время, когда новые научные данные показывают, что космический вакуум - это не пустота, гипотеза А.А. Белопольского приобретает вполне определённый физический смысл. Если вакуум - это материальная среда, то первое возражение не имеет силы. При прохождении света через вакуум как материальную среду фотоны должны терять энергию подобно тому, как это происходит при прохождении света через вещество. Этот механизм известен и описан в научной литературе[см., например, 13, c. 228]. Его суть состоит в следующем. В спектре рассеянного света помимо несмещённой спектральной линии содержатся новые линии, частоты которых представляют собой суммы или разности частоты падающего света $v$ и частоты собственных колебаний молекул рассеивающей среды $v_{\mathrm{e}}$. Согласно теории, каждой смещенной линии частоты $v-v_{\text {e }}$ (так называемой стоксовой компоненте) соответствует линия частоты $v+v_{\text {e }}$ (антистоксова компонента). Интенсивность антистоксовых линий значительно меньше, чем интенсивность стоксовых линий, особенно при низких температурах, что объясняет смещение частоты в сторону её уменьшения, т.е. красное смещение. Вакуумные пары можно рассматривать как молекулы рассеивающей среды.

Обратимся ко второму возражению. Действительно, если бы фотоны на своём пути встречали свободные частицы, то их направление должно было бы изменяться вследствие комптоновского рассеивания. Тогда изображения далёких галактик действительно казались бы размытыми. Но вакуум состоит не из отдельных 
свободных частиц, а из вакуумных пар. При прохождении фотонов через вакуум происходит их поглощение вакуумными парами с последующим излучением, подобно поглощению и излучению фотонов молекулами вещества. В силу закона сохранения импульса направления поглощенного и излученного фотонов должны совпадать. Следовательно, направление движения фотонов остаётся неизменным, а их энергия уменьшается вследствие возбуждения вакуумных пар.

Красные смещения в спектрах галактик рассматриваются как наблюдательная основа современной космологии (теории расширяющейся Вселенной). Принято считать, что эти смещения обусловлены взаимным удалением (разбеганием) галактик, т.е. имеют доплеровскую природу. Согласно прави лам формальной логики, утверждение о разбегании галактик можно считать истинным, если являются истинными два более простых высказывания:

1) разбегание галактик теоретически обосновано;

2) разбегание галактик подтверждается данными наблюдений.

Рассмотрим положение о доплеровской интерпретации красных смещений (разбегания галактик) с позиций формальной логики. Теоретическую основу гипотезы разбегания галактик составляет ОТО. Тогда первое высказывание можно считать истинным, если являются истинными следующие высказывания:

1) разбегание галактик следует из ОТ0;

2) ОТО является правильной теорией.

Как следует из решений уравнений ОТО, Вселенная может быть расширяющейся, сжимающейся и стационарной. Таким образом, разбегание галактик не противоречит ОТО, но и не следует однозначно из теории. Что касается утверждения о правильности ОТО, то здесь существуют различные мнения. Многие учёные считают, что ОТО не имеет достаточного для фундаментальной теории экспериментального подтверждения. Как отмечает Леон Бриллюэн (Brillouin, Leon Nicolas, 1889-1969), «нет никаких экспериментальных фактов, подтверждающих громоздкую в математическом отношении теорию Эйнштейна» [14, с. 83].

Итак, утверждение, что разбегание галактик теоретически обосновано, нельзя считать истин- ным. Оно основано на двух гипотезах, дополняющих одна другую. Тогда основным аргументом в пользу гипотезы разбегания галактик остаётся второе утверждение: разбегание галактик подтверждается данными наблюдений. Оно основано на установленной Хабблом закономерности «скорость-расстояние».

\section{обобщение и анализ данных наблюдений}

Поскольку «разбегание» галактик однозначно не следует из ОТО, то основным аргументом в пользу гипотезы расширения Вселенной можно считать только установленную Э. Хабблом закономерность «скорость-расстояние» (см. рис.1). Поэтому необходим объективный анализ, как самой закономерности, так и данных, которые ей противоречат.

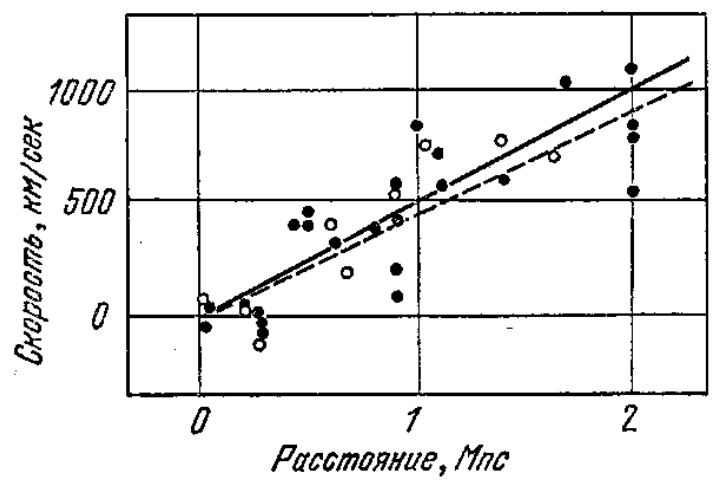

Рис.1. Диаграмма Хаббла*

* Зависимость лучевая скорость - расстояние до галактики. Пунктирная линия - по данным 1929 г., сплошная линия - по данным 1936 г.

В результате обобщения данных наблюдений определена зависимость «скорость-расстояние» (закон Хаббла):

$$
v=H \cdot r
$$

где $v$ - скорость удаления объекта, $r$ - расстояние до объекта, $H$ - постоянная Хаббла.

Как видно, полученную Э. Хабблом зависимость, представленную на диаграмме, лишь условно можно считать законом. Много точек находится далеко от интерполяционной прямой. Не удивительно, что постоянная Хаббла время от времени уточняется: её первона- 


\section{Исследования космоса 4(5) • 2017}

чальное значение составляло 500 км/(с·Мпк), в настоящее время оно находится в диапазоне $H=100 \div 50$ км/(с·Мпк).

Как в диаграмме, построенной Э. Хабблом, так и в более поздних диаграммах, наблюдаются существенные отклонения от прямолинейной зависимости «скорость-расстояние». Но главным тестом для проверки этой зависимости являются данные об объектах, которые не только находятся рядом на небесной сфере, но и имеют признаки общего происхождения. Необходим анализ наблюдательных данных о подобных объектах для выявления случаев, которые явно выпадают из закономерности «скорость-расстояние». Таких данных накопилось достаточно. К ним относятся сильно отличающиеся красные смещения, наблюдаемые в спектрах некоторых кратных галактик и спектрах парных объектов типа галактика-квазар.

На различные красные смещения объектов, принадлежащих одной группе, обратил внимание Борис Александрович Воронцов-Вельяминов (1902-1994), который привел ряд характерных примеров. Например, в тесном квинтете Стефана (рис. 2.) у четырех галактик красные смещения заключены в пределах 5920 - 7010 км/сек, а у пятого члена группы - галактики NGC 7520 смещение составляет 1070 км/сек, хотя раньше по положению, яркости и размеру не было сомнений, что эта галактика входит в ту же группу $[15$, c. 364].

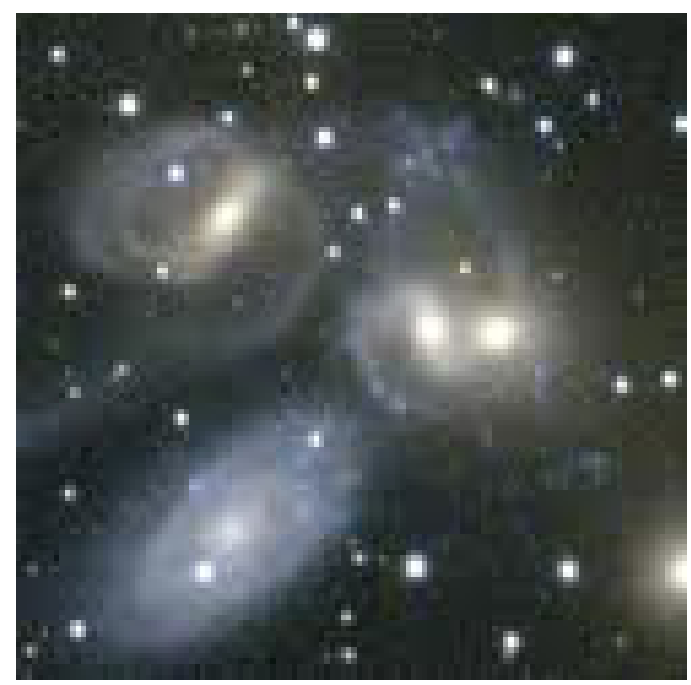

Рис. 2. Квинтет Стефана
В области Девы галактика IC 3481 и очень близкая к ней соседка имеют скорости 7086 и 7304 км/сек, причём обе галактики соединены перемычкой, т.е. являются взаимодействующими, и их физическая связь несомненна. Но от этой пары отходит очень длинный волнистый хвост, на конце которого находится галактика IC 3483 , по размеру и яркости очень похожая на IC 3481. Однако её красное смещение + 33 км/сек хотя и мало, но не исключает её принадлежности к скоплению Девы. Разность красных смещений более 7000 км/сек в группе галактик не только очень близких взаимно, но как будто связанных даже перемычкой, тут встретилась впервые. Случайность проекции IC 3483 на конец небывало длинного хвоста другой пары крайне маловероятна [15, с. 363].

Американский астроном Хэлтон Арп (Arp, Halton Christian, 1927-2013) объясняет происхождение больших космологических красных смещений как следствие процессов, происходящих в космических объектах. Он обнаружил ряд систем, связанных еле заметными струями, с объектами, имеющими различные красные смещения. Х. Арп представил эти объекты как доказательство того, что красное смещение обусловлено внутренней структурой изучаемых объектов, а не расстояниями до них [16].

Обстоятельные исследования аномальных красных смещений в спектрах квазаров и галактик с учетом обобщения результатов многих авторов были проведены индийским астрофизиком Джайянтом Нарликаром (Narlikar, Jayant Vishnu, род. 1938). Он обращает внимание на дополнительные результаты, которые трудно объяснить, исходя из случайного совпадения направлений на квазар и галактику. На рис. 3 проиллюстрированы особенности в расположении некоторых групп галактик и квазаров. Непонятно, почему квазары располагаются крест-накрест около галактики, если их расположение случайно. И, наконец, совершенно невозможно объяснить попарное совпадение красных смещений квазаров, если исходить из космологического происхождения красного смещения. Видимо, никак нельзя избежать вывода о том, что квазары с большими красными смещениями связаны с галактиками, имеющими малые красные смещения. 


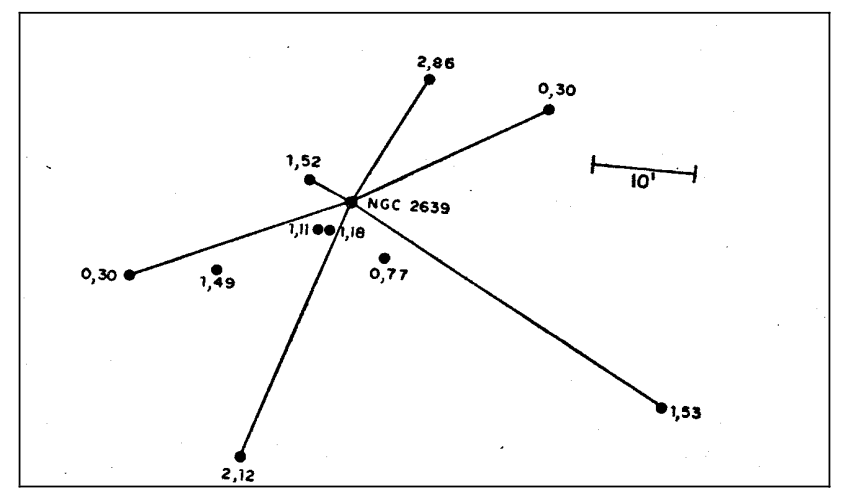

Рис. 3. Попарно-симметричное расположение квазаров с близкими значениями красного смещения около галактики NGC 2639 [17, с. 221].

Типичная ситуация такова: квазар $Q$ лежит на небесной сфере близко к галактике $G$, но их красные смещения, соответственно $z_{Q}$ и $z_{G}$, сильно различаются. При этом попарно симметричное расположение квазаров, как на рис. 3 , можно было бы объяснить тем, что квазары выбрасываются в противоположных направлениях. Но тут-то и возникает главная трудность. Если квазары вылетают из галактики во всех направлениях, то часть из них будет удаляться от нас (это объясняет большие красные смещения $z_{A}$ ), а часть будет приближаться к нам. Поэтому у этих последних должны наблюдаться большие синие смещения. Однако, как отмечает Нарликар, до сих пор ни у одного квазара не наблюдалось синего смещения. [17, с. 218-227].

В 1971 году супруги Бербидж (Burbidge, Geoffrey Ronald 1925-2010) и Бербидж (Burbidge, Eleanor Margaret, род. 1919) привели примеры пар галактика-квазар, у которых красные смещения сильно различаются, несмотря на то, что члены каждой пары расположены на небесной сфере очень близко друг к другу. Это даёт основание предположить, что они близки и в пространстве [17, с. 220] (см. табл. 1).

Итак, исключительно доплеровская интерпретация красных смещений (гипотеза разбегания галактик) противоречит данным многочисленных наблюдений. Различные красные смещения в спектрах галактик и других объектов, имеющих признаки совместного происхождения, могут быть обусловлены различной энергией излучаемых фотонов, и, следовательно, различными значениями потерянной ими энергии при движении в космическом пространстве. Тем самым, новые знания о вакууме как материальной среде и данные наблюдений подтверждают гипотезу А.А. Белопольского, которая не получила признания в силу существующих представлений о космическом вакууме как пустом пространстве.

Таким образом, можно предположить, что закон Э. Хаббла как наблюдательная основа теории расширяющейся Вселенной, вошел в противоречие с данными эмпирических наблюдений. В настоящее время известны такие космические объекты и наблюдаются такие процессы, которые не были известны в начале XX столетия, когда А. Эйнштейн и А.А. Фридман стали применять уравнения ОТО для описания Вселенной в целом. Эти процессы характеризуются бурной космогонической активностью и перемещением огромных масс вещества и энергии в локальных областях пространства, где действуют различные физические факторы, в том числе пока недостаточно изученные. Таким образом, объединив разнообразные физические факторы в

таблица 1

\section{Пары галактика-квазар с несовпадающими красными смещениями}

\begin{tabular}{|c|c|c|c|c|}
\hline Квазар $\boldsymbol{Q}$ & Галактика $\boldsymbol{G}$ & $\mathbf{z}_{\boldsymbol{Q}}$ & $\mathbf{z}_{\boldsymbol{G}}$ & $\boldsymbol{\vartheta}$, угл. мин \\
\hline 3C 232 & NGC 3067 & 0,534 & 0,0050 & 1,9 \\
\hline 3C 268.4 & NGC 4138 & 1,400 & 0,0036 & 2,9 \\
\hline 3C 275.1 & NGC 4651 & 0,557 & 0,0025 & 3,5 \\
\hline 3C 309.1 & NGC 5832 & 0,904 & 0,0020 & 6,2 \\
\hline 3C 455 & NGC 7413 & 0,543 & 0,0332 & 0,4 \\
\hline
\end{tabular}




\section{Исследования космоса 4(5) • 2017}

единый тензор энергии-импульса, приверженцы космологии Эйнштейна-Фридмана на основании отдельных наблюдательных данных о красных смещениях в спектрах галактик заранее предопределили геометрию мира в целом, не установив достоверно причину, вызывающую эти красные смещения.

\section{Заключение}

В заключение следует привести высказывание Л. Бриллюэна о теории расширяющейся Вселенной: «некоторые традиционные науки представляют собой странную смесь наблюдений и их интерпретации, основывающейся на отличных теориях, но экстраполируемой столь далеко за пределы условий эксперимента, что невольно настораживаешься и удивляешься: как часто желаемое выдаётся за действительное и тщательный анализ подменяется фантазированием. Приятно рассуждать о происхождении Вселенной, но надо помнить, что такие рассуждения - лишь чистая фантазия» [14, с. 17].

В настоящей работе получила дальнейшее развитие теоретическая модель физического (космического) вакуума как формы материи, ранее изложенная в $[1,2]$. Было рассмотрено приложение данной модели к новой интерпретации красных смещений в спектрах галактик, отличной от принятой настоящее время доплеровской интерпретации.

Большинство специалистов считает, что красные смещения имеют доплеровскую природу, т.е. обусловлены скоростью удаления галактик, как это следует из закона Хаббла. Разбегание галактик (расширение Вселенной) является исходным положением современной космологии.
Однако следует отметить, что скорость удаления галактик не является наблюдаемой величиной, а вычисляется, исходя из гипотезы доплеровской природы красных смещений. Формирование современной космологии происходило в основном в первой трети XX столетия на базе ограниченных знаний в этой области, которыми тогда располагала наука.

К настоящему времени объем знаний в этой области существенно возрос. Однако космология продолжает развиваться в рамках устаревших представлений, игнорируя противоречащие ей новые данные наблюдений. Теперь, когда мы знаем, что космическое пространство не является пустотой, красное смещение можно объяснить как эффект взаимодействия фотонов с космическим вакуумом. В свете современных знаний о вакууме гипотеза доплеровской природы красных смещений представляется неполной и устаревшей. В её основе лежит представление о космическом вакууме как пустоте. Это противоречит новым опытным данным, а именно, вакуумным эффектам квантовой теории поля и существованию квантовых жидкостей со свойствами, подобным свойствам физического вакуума, изучаемых в физике конденсированных сред.

Целесообразно рассматривать красные смещения в спектрах галактик как вакуумный эффект, обусловленный потерей энергии фотонами при их прохождении через вакуум как материальную среду. Такой подход позволяет на основе новых научных данных вернуться к ранее отвергнутой гипотезе известного российского и советского астрофизика А.А. Белопольского о потере энергии фотонами при их движении в космическом пространстве [3, с. 266-267].

\section{Библиография}

1. Серга Э. В. Физический вакуум как форма материи: новый взгляд на структуру и свойства // Исследования космоса. 2017. №2. С. 85-100.

2. Серга Э. В. Космический вакуум как материальная среда // Исследования космоса. 2017. №3. C. $164-172$.

3. Белопольский А. А. Астрономические труды. М.- Л.: Гостехиздат, 1954.

4. Максвелл Дж. Трактат об электричестве и магнетизме / Серия «Классики науки». М.: Наука, 1989. 
5. Воловик Г.Е. От эфира Ньютона к вакууму современной физики конденсированных сред // Ньютон и философские проблемы ХХ века. - М.: Наука, 1991. С. 88-98.

6. Эйнштейн А. Теория относительности // Собр. Научных трудов. T.I. - М.: Наука, 1965. С. 175-186.

7. Эйнштейн А. Эфир и теория относительности // Собр. Научных трудов. T.I. - М.: Наука, 1965. C. 682-689.

8. Эйнштейн А. Вопросы космологии и общая теория относительности // Собр. Научных трудов. T.I. - М.: Наука, 1965. С. 691-612.

9. Фридман А. А. Избранные труды. - М.: Наука, 1966.

10. Эйнштейн А. Замечание о работе А. Фридмана «О кривизне пространства» // Собр. Научных трудов. Т.2. - М.: Наука, 1966. С. 118

11. Эйнштейн А. К работе А. Фридмана «О кривизне пространства» // Собр. Научных трудов. Т.2. - М.: Наука, 1966. С. 119.

12. Эйнштейн А. К космологической проблеме общей теории относительности // Собр. Научных трудов. Т.2. - М.: Наука, 1966. С. 349-352.

13. Физика микромира: маленькая энциклопедия / гл. ред. чл.-корр. АН СССР Д.В. Ширков. М.: Советская энциклопедия, 1980.

14. Бриллюэн Л. Новый взгляд на теорию относительности. Пер. с англ. - М.: Мир, 1972. - 143 с.

15. Воронцов-Вельяминов Б. А. Внегалактическая астрономия. - М.: Наука, 1978. - 480 с.

16. Arp H. Peculiar Galaxies and Radio Sources // Astrophysical Journal. 1967. Vol. 148. P. 321-366.

17. Нарликар Дж. Неистовая Вселенная. Пер. с англ. М.: Мир, 1985. - 256 с.

\section{References (transliterated)}

1. Serga E. V. Fizicheskii vakuum kak forma materii: novyi vzglyad na strukturu i svoistva // Issledovaniya kosmosa. 2017. №2. S. 85-100.

2. Serga E. V. Kosmicheskii vakuum kak material'naya sreda // Issledovaniya kosmosa. 2017. №3. S. 164-172.

3. Belopol'skii A. A. Astronomicheskie trudy. M.- L.: Gostekhizdat, 1954.

4. Maksvell Dzh. Traktat ob elektrichestve i magnetizme / Seriya «Klassiki nauki». M.: Nauka, 1989.

5. Volovik G.E. Ot efira N'yutona k vakuumu sovremennoi fiziki kondensirovannykh sred // N'yuton i filosofskie problemy KhKh veka. - M.: Nauka, 1991. S. 88-98.

6. Einshtein A. Teoriya otnositel'nosti // Sobr. Nauchnykh trudov. T.I. - M.: Nauka, 1965. S. 175-186.

7. Einshtein A. Efir i teoriya otnositel'nosti // Sobr. Nauchnykh trudov. T.I. - M.: Nauka, 1965. S. 682-689.

8. Einshtein A. Voprosy kosmologii i obshchaya teoriya otnositel'nosti // Sobr. Nauchnykh trudov. T.I. - M.: Nauka, 1965. S. 691-612.

9. Fridman A. A. Izbrannye trudy. - M.: Nauka, 1966.

10. Einshtein A. Zamechanie o rabote A. Fridmana «O krivizne prostranstva» // Sobr. Nauchnykh trudov. T.2. - M.: Nauka, 1966. S. 118

11. Einshtein A. K rabote A. Fridmana «O krivizne prostranstva» // Sobr. Nauchnykh trudov. T.2. - M.: Nauka, 1966. S. 119.

12. Einshtein A. K kosmologicheskoi probleme obshchei teorii otnositel'nosti // Sobr. Nauchnykh trudov. T.2. - M.: Nauka, 1966. S. 349-352.

13. Fizika mikromira: malen'kaya entsiklopediya / gl. red. chl.-korr. AN SSSR D.V. Shirkov. M.: Sovetskaya entsiklopediya, 1980.

14. Brillyuen L. Novyi vzglyad na teoriyu otnositel'nosti. Per. s angl. - M.: Mir, 1972. - 143 s.

15. Vorontsov-Vel'yaminov B. A. Vnegalakticheskaya astronomiya. - M.: Nauka, 1978. - 480 s.

16. Arp H. Peculiar Galaxies and Radio Sources // Astrophysical Journal. 1967. Vol. 148. P. 321-366.

17. Narlikar Dzh. Neistovaya Vselennaya. Per. s angl. M.: Mir, 1985. - 256 s. 